\title{
ON PROFINITE GROUPS IN WHICH COMMUTATORS ARE ENGEL
}

\author{
PAVEL SHUMYATSKY
}

(Received 26 February 1999; revised 29 February 2000)

Communicated by R. B. Howlett

\begin{abstract}
We show that if $G$ is a finitely generated profinite group such that $\left[x_{1}, x_{2}, \ldots, x_{k}\right]$ is Engel for any $x_{1}, x_{2}, \ldots, x_{k} \in G$, then $\gamma_{k}(G)$ is locally nilpotent, and if $\left[x_{1}, x_{2}, \ldots, x_{k}\right]$ has finite order for any $x_{1}, x_{2}, \ldots, x_{k} \in G$ then, under some additional assumptions, $\gamma_{k}(G)$ is locally finite.
\end{abstract}

2000 Mathematics subject classification: primary 20E18, 20F40; secondary: $20 \mathrm{~F} 45$.

Keywords and phrases: profinite group, associated Lie algebra.

\section{Introduction}

The positive solution of the Restricted Burnside Problem [15, 16] had led to many remarkable results on profinite groups. In particular, using Wilson's reduction theorem [12], Zel'manov has been able to prove local finiteness of periodic compact groups [17], thus answering positively a major problem. Another result of this nature is that any profinite Engel group is locally nilpotent [14]. In the present paper we obtain some further corollaries to the solution of the Restricted Burnside Problem. Our main results generalize, fully or partially, the above cited ones.

Let $\mathscr{C}$ be a class of groups. A pro- $\mathscr{C}$ group is a topological group that is isomorphic to an inverse limit of groups in $\mathscr{C}$. Thus, a profinite group is exactly a pro- $\mathscr{C}$ group with $\mathscr{C}$ being the class of all finite groups and a pro- $p$ group is that with $\mathscr{C}$ being the class of all finite $p$-groups for a fixed prime $p$ (see [1] for basic properties of profinite and pro- $p$ groups). For a positive integer $h$, let $\mathscr{N}^{h}$ denote the class of finite soluble groups $G$ of Fitting height $h(G)$ at most $h$. Obviously, a profinite group $G$ is a pro- $\mathscr{N}^{h}$ group if and only if $G / N$ is soluble with $h(G / N) \leq h$ for any open

(C) 2001 Australian Mathematical Society 0263-6115/2001 \$A2.00+0.00 
normal subgroup $N$ of $G$. We will use the term 'pro-(finite nilpotent) group' rather than 'pro- $\mathscr{N}$ group'.

Let $G$ be any group and let $k, n$ be positive integers. For $x, y \in G$ we use $[x, y]$ to denote the group-commutator $x^{-1} y^{-1} x y$. The long commutators $\left[x_{1}, x_{2}, \ldots, x_{k}\right]$ and $\left[x,{ }_{n} y\right]$ are defined inductively by

$$
\left[x_{1}\right]=x_{1} ; \quad\left[x_{1}, x_{2}, \ldots, x_{k}\right]=\left[\left[x_{1}, x_{2}, \ldots, x_{k-1}\right], x_{k}\right]
$$

and

$$
[x, 0 y]=x ; \quad\left[x,{ }_{n} y\right]=\left[\left[x,{ }_{n-1} y\right], y\right] .
$$

An element $y \in G$ is called Engel if for any $x \in G$ there exists a number $n$ such that $\left[x,{ }_{n} y\right]=1$. A group $G$ is Engel if any element of $G$ is Engel. By the mentioned above result of Wilson and Zel'manov a profinite Engel group is locally nilpotent. In this paper we prove the following result.

THEOREM 1.1. Let $k$ be a positive integer, $G$ a finitely generated profinite group such that $\left[x_{1}, x_{2}, \ldots, x_{k}\right]$ is Engel for any $x_{1}, x_{2}, \ldots, x_{k} \in G$. Then $\gamma_{k}(G)$ is locally nilpotent.

A subset $X$ of a topological group $G$ is said to generate a subgroup $H$ if $H$ is the smallest closed subgroup of $G$ that contains $X$. As usual, $\gamma_{k}(G)$ stands for the smallest normal closed subgroup $N$ of $G$ such that $G / N$ is nilpotent of class at most $k-1$. It is well known that $\gamma_{k}(G)$ is generated by the set $\left\{\left[x_{1}, \ldots, x_{k}\right] ; x_{1}, \ldots, x_{k} \in G\right\}$.

We also investigate profinite groups in which all commutators $\left[x_{1}, \ldots, x_{k}\right]$ have finite order. The following conjecture seems to be plausible.

CONJECTURE. Let $k$ be a positive integer, $G$ a finitely generated profinite group such that $\left[x_{1}, x_{2}, \ldots, x_{k}\right]$ is of finite order for any $x_{1}, x_{2}, \ldots, x_{k} \in G$. Then $\gamma_{k}(G)$ is locally finite.

Our next result is an evidence in favour of the above conjecture.

THEOREM 1.2. Let $k$ and $h$ be positive integers, $G$ a finitely generated pro- $\mathscr{N}^{h}$ group such that $\left[x_{1}, x_{2}, \ldots, x_{k}\right]$ is of finite order for any $x_{1}, x_{2}, \ldots, x_{k} \in G$. Then $\gamma_{k}(G)$ is locally finite.

The techniques used in this paper are, by and large, the same as in [14]. In particular, we make heavy use of the so called Lie ring methods. For the reader's convenience we collect in the next section some necessary definitions and facts on Lie algebras associated with pro- $p$ groups. The most adequate general reference for the subject is [18]. 


\section{Associated Lie algebras}

Let $L$ be a Lie algebra over a field $K$. We use the left normed notation: thus if $l_{1}, l_{2}, \ldots, l_{n}$ are elements of $L$, then

$$
\left[l_{1}, l_{2}, \ldots, l_{n}\right]=\left[\ldots\left[\left[l_{1}, l_{2}\right], l_{3}\right], \ldots, l_{n}\right]
$$

An element $a \in L$ is called ad-nilpotent if there exists a positive integer $n$ such that $\left[x,{ }_{n} a\right]=0$ for all $x \in L$. If $n$ is the least integer with the above property, then we say that $a$ is ad-nilpotent of index $n$. Let $X \subseteq L$ be any subset of $L$. By a commutator in elements of $X$ we mean any element of $L$ that could be obtained from elements of $X$ by means of repeated operation of commutation with an arbitrary system of brackets including the elements of $X$. Denote by $F$ the free Lie algebra over $K$ on countably many free generators $x_{1}, x_{2}, \ldots$ Let $f=f\left(x_{1}, x_{2}, \ldots, x_{n}\right)$ be a non-zero element of $F$. The algebra $L$ is said to satisfy the identity $f=0$ if $f\left(a_{1}, a_{2}, \ldots, a_{n}\right)=0$ for any $a_{1}, a_{2}, \ldots, a_{n} \in L$. In this case we say that $L$ is PI. We are now in a position to quote a theorem of Zel'manov [18, III(0.4)] which has numerous important applications to group theory.

THEOREM 2.1. Let $L$ be a Lie algebra generated by $a_{1}, a_{2}, \ldots, a_{m}$. Assume that $L$ is $P I$ and that each commutator in the generators $a_{1}, a_{2}, \ldots, a_{m}$ is ad-nilpotent. Then $L$ is nilpotent.

Let $G$ be a group. We call an element $x \in G$ a simple commutator of weight $k$ if there exist $x_{1}, \ldots, x_{k} \in G$ such that $x=\left[x_{1}, \ldots, x_{k}\right]$. Let us note that if $x \in G$ is a simple commutators of weight $k$, then so is $[x, y]$ for any $y \in G$. This elementary observation will play an important rôle in the paper.

Fixed a prime $p$, we denote by $D_{i}=D_{i}(G)$ the $i$-th dimension subgroup of $G$ in characteristic $p$. These subgroups form a central series of $G$ known as the ZassenhausJennings-Lazard series. Set $L(G)=\oplus D_{i} / D_{i+1}$. Then $L(G)$ can naturally be viewed as a Lie algebra over the field $\mathscr{F}_{p}$ with $p$ elements. The subalgebra of $L$ generated by $D_{1} / D_{2}$ will be denoted by $L_{p}(G)$. The following lemma is implicit in [18, page 71].

LEMMA 2.2. Let $G$ be a pro-p group generated by $g_{1}, g_{2}, \ldots, g_{m}$. For a positive integer $c$ let $\rho_{1}, \rho_{2}, \ldots, \rho_{r}$ be the list of all simple commutators in $g_{1}, g_{2}, \ldots, g_{m}$ of weight at most $c$ (so $r$ obviously is bounded by a function of $m$ and $c$ ). If $L_{p}(G)$ is nilpotent of class $c$, then $G=\left\langle\rho_{1}\right\rangle\left\langle\rho_{2}\right\rangle \cdots\left\langle\rho_{r}\right\rangle$ is the product of procyclic subgroups generated by the $\rho_{i}$ 's. In particular, if each $\rho_{i}$ has finite order, then $G$ is finite.

Another corollary of nilpotency of $L_{p}(G)$ is the Lazard criterion for a pro-p group to be $p$-adic analytic [6]. 
THEOREM 2.3. Let $G$ be a finitely generated pro-p group. If $L_{p}(G)$ is nilpotent, then $G$ is $p$-adic analytic.

Let $x \in G$, and let $i=i(x)$ be the largest integer such that $x \in D_{i}$. We denote by $\tilde{x}$ the element $x D_{i+1} \in L(G)$. We now cite two results providing sufficient conditions for $\tilde{x}$ to be ad-nilpotent. The following lemma is immediate from the proof of [14, Lemma 3].

LEMMA 2.4. Let $x$ be an Engel element of a profinite group $G$. Then $\tilde{x}$ is adnilpotent.

LEMMA 2.5 (Lazard, [5, page 131]). For any $x \in G$ we have $(\text { ad } \tilde{x})^{p}=$ ad $\left(\tilde{x}^{p}\right)$. In particular, if $x^{q}=1$, then $\tilde{x}$ is ad-nilpotent of index at most $q$.

Let $H$ be a subgroup of $G$ and $a_{1}, \ldots, a_{n} \in G$. Let $w=w\left(x_{1}, \ldots, x_{n}\right)$ be a nontrivial element of the free group on the set $\left\{x_{1}, \ldots, x_{n}\right\}$. Following [14] we say that the law $w=1$ is satisfied on the cosets $a_{1} H, \ldots, a_{n} H$ if $w\left(a_{1} h_{1}, \ldots, a_{n} h_{n}\right)=1$ for any $h_{1}, \ldots, h_{n} \in H$. In [14] Wilson and Zel'manov proved the following theorem.

THEOREM 2.6. If $G$ is a group which has a subgroup $H$ of finite index and elements $a_{1}, \ldots, a_{n}$ such that a law $w=1$ is satisfied on the cosets $a_{1} H, \ldots, a_{n} H$, then for each prime $p$ the Lie algebra $L_{p}(G)$ is $P I$.

\section{A Hartley type result}

In this section we prove that if $G$ is a finitely generated pro- $\mathscr{N}^{h}$ group, then every element of $\gamma_{k}(G)$ can be expressed as a product of finitely many simple commutators of weight $k$. This result was obtained by Hartley for $k=2$ [2] and the general case actually follows from the argument in [2]. For the reader's convenience we describe the proof.

LEMMA 3.1. Let $n \geq 1$ and $k \geq 2$. Let $G$ be a group, $a_{1}, \ldots, a_{n}, g \in G$ and assume that each $a_{i}$ is a simple commutator of weight $k-1$. Then $\left[a_{1} \cdots a_{n}, g\right]$ can be expressed as a product of at most $2 n-1$ simple commutators of weight $k$.

PROOF. This is quite straightforward by induction on $n$. If $n=1$ the result is obvious. If $n \geq 2$ we write

$$
\left[a_{1} \cdots a_{n}, g\right]=\left[a_{1}, g\right]\left[a_{1}, g, a_{2} \cdots a_{n}\right]\left[a_{2} \cdots a_{n}, g\right]
$$

and use the induction hypothesis. 
THEOREM 3.2. Let $G$ be an m-generated finite soluble group of Fitting height $l$. There exists a constant $N(m, k, l)$ depending only on $m, k$ and $l$ such that each element of $\gamma_{k}(G)$ can be expressed as a product of at most $N(m, k, l)$ simple commutators of weight $k$.

Proof. It is sufficient to deal only with the case $k \geq 2$. Assume first that $G$ is nilpotent and suppose by induction that the number $N(m, k-1,1)$ has already been found. Set $H=\gamma_{k-1}(G)$. Obviously there exists a number $u=u(k, m)$ depending only on $k$ and $m$ such that $H=\left\langle y_{1}^{G}, \ldots, y_{u}^{G}\right\rangle$ for suitably chosen $y_{1}, \ldots, y_{u} \in H$. Therefore, by [2, Lemma 3], every element $x$ of $\gamma_{k}(G)=[H, G]$ can be written in the form

$$
x=\left[h_{1}, g_{1}\right] \cdots\left[h_{m+u}, g_{m+u}\right],
$$

where $h_{i} \in H, g_{i} \in G$. By the induction hypothesis each $h_{i}$ can be written as a product of at most $N(m, k-1,1)$ simple commutators of weight $k-1$. Now Lemma 3.1 says that each commutator $\left[h_{i}, g_{i}\right]$ can be written as a product of boundedly many simple commutators of weight $k$. This establishes the claim in the case $l=1$.

Suppose now that $l \geq 2$ and the number $N(m, k, l-1)$ has been found. Let $R$ be the intersection of all normal subgroups $N$ of $G$ such that $h(G / N)=l-1$. Then, of course, $R$ is nilpotent, $h(G / R)=l-1$ and $R=[R, G]$. In particular, $R \leq \gamma_{j}(G)$ for any $j \geq 1$. Arguing like Hartley in the proof of [2, Theorem 2] we conclude that there exist a number $t=t(m, l)$ depending only on $m$ and $l$ and elements $r_{1}, \ldots, r_{t} \in R$ such that $R=\left\langle r_{1}^{G}, \ldots, r_{t}^{G}\right\rangle$. Therefore, by [2, Lemma 3], every element $x$ of $[R, G]=R$ can be written in the form

$$
x=\left[h_{1}, g_{1}\right] \cdots\left[h_{m+t}, g_{m+t}\right]
$$

where $h_{i} \in R, g_{i} \in G$. Since each $h_{i} \in \gamma_{k-1}(G)$, by the induction hypothesis we can write $h_{i}=a_{i 1} \cdots a_{i n}$, where $n=N(m, k-1, l)$ and $a_{i 1}, \ldots, a_{i n}$ are simple commutators of weight $k-1$. Substituting now in (*) each $h_{i}$ by $a_{i 1} \cdots a_{i n}$ and applying Lemma 3.1 we obtain that any element in $R$ can be expressed as a product of boundedly many simple commutators of weight $k$. Taking into account that $h(G / R)=l-1$ and applying the induction hypothesis to $G / R$, the result follows.

The next corollary is now immediate.

COROLLARY 3.3. Let $k, h \geq 1$ and let $G$ be a finitely generated pro- $\mathscr{N}^{h}$ group. Then every element of $\gamma_{k}(G)$ can be expressed as a product of finitely many simple commutators of weight $k$. 


\section{Proof of the main results}

We establish Theorem 1.1 and Theorem 1.2 using the following related results on residually finite groups (see $[8,9,10])$.

THEOREM 4.1. Let $k, n \geq 1$. Let $G$ be a residually finite group such that $\left[y,{ }_{n}\left[x_{1}\right.\right.$, $\left.\left.x_{2}, \ldots, x_{k}\right]\right]=1$ for any $y, x_{1}, x_{2}, \ldots, x_{k} \in G$. Then $\gamma_{k}(G)$ is locally nilpotent.

THEOREM 4.2. Let $q$ be a prime-power and $k \geq 1$. Let $G$ be a residually finite group such that $\left[x_{1}, x_{2}, \ldots, x_{k}\right]^{q}=1$ for any $x_{1}, x_{2}, \ldots, x_{k} \in G$. Then $\gamma_{k}(G)$ is locally finite.

It is convenient to prove first Theorem 1.1 and Theorem 1.2 under the hypothesis that $G$ is pro-(finite nilpotent) and then extend the results to the general case.

PROPOSITION 4.3. Let $G$ be a finitely generated pro-(finite nilpotent) group such that $\left[x_{1}, x_{2}, \ldots, x_{k}\right]$ is Engel for any $x_{1}, x_{2}, \ldots, x_{k} \in G$. Then $\gamma_{k}(G)$ is locally nilpotent.

PROOF. Set $\bar{G}=G \times G \times \cdots \times G(k+1$ factors $)$. For each integer $n$ we set

$$
S_{n}=\left\{\left(b, a_{1}, \ldots, a_{k}\right) \in \bar{G} ;\left[b,{ }_{n}\left[a_{1}, \ldots, a_{k}\right]\right]=1\right\} .
$$

Since the sets $S_{n}$ are closed in $\bar{G}$ and cover $\bar{G}$, by Baire's category theorem [4, page 200] there exist an open subgroup $H$ of $G$, elements $b, a_{1}, \ldots, a_{k} \in G$ and an integer $n$ such that the cosets $b H, a_{1} H, \ldots, a_{k} H$ satisfy the law $\left[y,{ }_{n}\left[x_{1}, \ldots, x_{k}\right]\right]=1$. Let $|G: H|=m$ and write $K$ for the product of the Sylow subgroups $P_{1}, \ldots, P_{r}$ of $G$ corresponding to the primes dividing $m$. Since $G \cong P_{1} \times P_{2} \times \cdots \times P_{r} \times G / K$ and since $G=P_{1} \cdots P_{r} H$, it follows that $G / K$ satisfies the law $\left[y,{ }_{n}\left[x_{1}, \ldots, x_{k}\right]\right]=1$. Hence $\gamma_{k}(G / K)$ is locally nilpotent by Theorem 4.1 . It therefore suffices to show that $\gamma_{k}\left(P_{i}\right)$ is locally nilpotent for any $i=1, \ldots, r$ and so we assume that $G$ is a pro- $p$ group. Since any element of $\gamma_{k}(G)$ can be expressed as a product of finitely many simple commutators weight $k$, it is sufficient to show that any abstract subgroup $Q$ generated by finitely many simple commutators $a_{1}, \ldots, a_{s}$ of weight $k$, is nilpotent. Let $\bar{Q}$ be the closure of $Q$. By Theorem $2.6 L=L_{p}(\bar{Q})$ is PI. Let $\tilde{a}_{1}, \ldots, \tilde{a}_{s}$ be the homogeneous elements of $L$ corresponding to $a_{1}, \ldots, a_{s}$. Since any group commutator in $a_{1} \ldots, a_{s}$ is Engel, Lemma 2.4 shows that any Lie commutator in $\tilde{a}_{1}, \ldots, \tilde{a}_{s}$ is ad-nilpotent. Zel'manov's Theorem 2.1 now tells us that $L$ is nilpotent. Therefore, $\bar{Q}$ is $p$-adic analytic (Lemma 2.3). Obviously $\bar{Q}$ cannot contain a subgroup isomorphic to the free discrete group of rank two, so by the Tits' Alternative [11] $\bar{Q}$ has a soluble subgroup of finite index. The claim now follows from a result of Plotkin [7, Theorem 7.14]. 
PROOF OF THEOREM 1.1. Since all Engel elements of a finite group lie in the Fitting subgroup [3, III, 6.14], it follows that $\gamma_{k}(K)$ is nilpotent for any finite quotient $K$ of $G$. Hence $\gamma_{k}(G)$ is pro-(finite nilpotent) and by Corollary 3.3 each element of $\gamma_{k}(G)$ can be expressed as a product of finitely many simple commutators $\left[x_{1}, x_{2}, \ldots, x_{k}\right]$ of weight $k$. It is therefore sufficient to show that any abstract subgroup $H$ generated by finitely many simple commutators of $a_{1}, \ldots, a_{s}$ of weight $k$ is nilpotent. Let $\bar{H}$ be the closure of $H$. We already know that $\bar{H}$ is pro-(finite nilpotent) and so the above proposition shows that $\gamma_{k}(\bar{H})$ is locally nilpotent. Obviously this implies that $\gamma_{k}(H)$ is locally nilpotent and the claim follows from a result of Plotkin [7, Theorem 7.14] because $H$ is generated by Engel elements.

To prove Theorem 1.2 some more preparatory work is required. The following lemma is due to Wilson [13].

LEMMA 4.4. Let $G$ be a finitely generated residually finite-nilpotent group. For each prime $p$ let $J_{p}$ denote the intersection of all normal subgroups of $G$ having finite $p$-power index. Assume that $G / J_{p}$ is nilpotent for any $p$. Then $G$ is nilpotent.

LEMMA 4.5. Let $G$ be a residually finite group such that $\left[x_{1}, x_{2}, \ldots, x_{k}\right]^{n}=1$. Assume that there exists $h \geq 1$ such that any finite quotient of $G$ is soluble and has Fitting height at most $h$. If $G$ is generated by finitely many elements of finite order, then $G$ is finite.

PROOF. If $G$ is residually- $p$ for some prime $p$, then, by Theorem $4.2, \gamma_{k}(G)$ is locally finite and we are done. If $G$ is residually nilpotent Lemma 4.4 allows us to reduce the problem to the 'residually- $p$ ' case.

Hence, we can assume that $h \geq 2$ and use induction on $h$. Let $R$ be the intersection of all normal subgroup $N$ of $G$ such that $G / N$ is nilpotent and finite. By the previous paragraph $R$ has finite index in $G$ and therefore $\gamma_{s}(G) \leq R$ for some $s$. Without any loss of generality we can assume that $s \geq k$. Obviously $\gamma_{s}(G)$ has finite index in $G$ and therefore $\gamma_{s}(G)$ is generated by finitely many elements of finite order. Furthermore, since $\gamma_{s}(G) \leq R$, it follows that $\gamma_{s}(G)$ is residually of Fitting height at most $h-1$. By the induction hypothesis we now derive that $\gamma_{s}(G)$ is finite, whence the lemma follows.

LEMMA 4.6. Let $n \geq 1, G$ a residually finite-nilpotent group such that $\left[x_{1}, x_{2}, \ldots\right.$, $\left.x_{k}\right]^{n}=1$ for any $x_{1}, x_{2}, \ldots, x_{k} \in G$. Then $\gamma_{k}(G)$ is locally finite.

ProOF. Let $J_{p}$ have the same meaning as in Lemma 4.4. Let $T$ denote the direct product $\prod G / J_{p}$, where $p$ ranges through the set of prime divisors of $n$. If $p$ is not a divisor of $n$ then $\gamma_{k}(G) \leq J_{p}$. Therefore, $\gamma_{k}(G)$ can be embedded in $\gamma_{k}(T)$. By Theorem $4.2 \gamma_{k}\left(G / J_{p}\right)$ is locally finite for any prime $p$ and the result follows. 
PROPOSITION 4.7. Let $G$ be a finitely generated pro-(finite nilpotent) group such that $\left[x_{1}, x_{2}, \ldots, x_{k}\right]$ is of finite order for any $x_{1}, x_{2}, \ldots, x_{k} \in G$. Then $\gamma_{k}(G)$ is locally finite.

PROOF. Set $\bar{G}=G \times G \times \cdots \times G$ ( $k$ factors). For each integer $n$ we set

$$
S_{n}=\left\{\left(a_{1}, \ldots, a_{k}\right) \in \bar{G} ;\left[a_{1}, \ldots, a_{k}\right]^{n}=1\right\} .
$$

Since the sets $S_{n}$ are closed in $\bar{G}$ and cover $\bar{G}$, by Baire's category theorem there exist an open subgroup $H$ of $G$, elements $a_{1}, \ldots, a_{k} \in G$ and an integer $n$ such that the cosets $a_{1} H, \ldots, a_{k} H$ satisfy the law $\left[x_{1}, \ldots, x_{k}\right]^{n}=1$. Let $|G: H|=m$ and write $K$ for the product of the Sylow subgroups $P_{1}, \ldots, P_{r}$ of $G$ corresponding to the primes dividing $m$. Since $G \cong P_{1} \times \cdots \times P_{r} \times G / K$ and since $G=P_{1} \cdots P_{r} H$, it follows that $G / K$ satisfies the law $\left[x_{1}, \ldots, x_{k}\right]^{n}=1$. Hence $\gamma_{k}(G / K)$ is locally finite by Lemma 4.6. It therefore suffices to show that $\gamma_{k}\left(P_{i}\right)$ is locally finite for any $i=1, \ldots, r$ and so we assume that $G$ is a pro- $p$ group. Since any element of $\gamma_{k}(G)$ can be expressed as a product of finitely many commutators of the form $\left[x_{1}, x_{2}, \ldots, x_{k}\right]$, it is sufficient to show that any abstract subgroup $Q$ generated by finitely many simple commutators of $a_{1}, \ldots, a_{s}$ of weight $k$ is finite. Let $\bar{Q}$ be the closure of $Q$. By Theorem $2.6 L=L_{p}(\bar{Q})$ is PI. Let $\tilde{a}_{1}, \ldots, \tilde{a}_{s}$ be the homogeneous elements of $L$ corresponding to $a_{1}, \ldots, a_{s}$. Since any group-commutator in $a_{1}, \ldots, a_{s}$ is of finite order, it follows from Lemma 2.5 that any Lie commutator in $\tilde{a}_{1}, \ldots, \tilde{a}_{s}$ is ad-nilpotent. Zel'manov's Theorem 2.1 now tells us that $L$ is nilpotent. Using Lemma 2.2 we therefore conclude that $Q$ is finite.

LEMMA 4.8. Let $G$ be a soluble profinite group generated by finitely many elements of finite order. If $\left[x_{1}, x_{2}, \ldots, x_{k}\right]$ is of finite order for any $x_{1}, x_{2}, \ldots, x_{k} \in G$, then $G$ is finite.

PROOF. The lemma is obvious if $k=1$ so assume that $k \geq 2$. We remark that $\gamma_{k}(G)$ has finite index in $G$ and therefore, by Corollary 3.3, $\gamma_{k}(G)$ is generated by finitely many elements of finite order. Arguing by induction on the derived length of $G$ and applying the induction hypothesis to $\gamma_{k}(G)$ in place of $G$ we conclude that $\gamma_{k}(G)$ is finite and the lemma follows.

Proof of THEOREM 1.2. By Proposition 4.7 we can assume that $h \geq 2$. Since, by Corollary 3.3, each element of $\gamma_{k}(G)$ can be expressed as a product of finitely many commutators of the form $\left[x_{1}, x_{2}, \ldots, x_{k}\right]$, it is sufficient to show that any abstract subgroup $H$ generated by finitely many simple commutators $a_{1}, \ldots, a_{s}$ of weight $k$ is finite. Let $\bar{H}$ be the closure of $H$ and $F$ a closed normal pronilpotent subgroup of $G$ such that $G / F$ is a pro- $N^{h-1}$ group. Arguing by induction on $h$ we can assume that 
$\gamma_{k}(G / F)$ is locally finite. Therefore, $F_{0}=F \cap \bar{H}$ has finite index in $\bar{H}$. Hence $F_{0}$ is finitely generated and Proposition 4.7 shows that $\gamma_{k}\left(F_{0}\right)$ is locally finite. Obviously $\gamma_{k}\left(F_{0}\right)$ is closed in $\bar{H}$. The above lemma shows that $\bar{H} / \gamma_{k}\left(F_{0}\right)$ is finite and this concludes the proof.

\section{References}

[1] J. D. Dixon, M. P. F. du Sautoy, A. Mann and D. Segal, Analytic p-adic groups (Cambridge University Press, Cambridge, 1991).

[2] B. Hartley, 'Subgroups of finite index in profinite groups', Math. Z. 168 (1979), 71-76.

[3] B. Huppert, Endliche Gruppen I (Springer, Berlin, 1967).

[4] J. L. Kelley, General topology (Van Nostrand, New York, 1955).

[5] M. Lazard, 'Sur les groupes nilpotents et les anneaux de Lie', Ann. Sci. École Norm. Supr. 71 (1954), 101-190.

[6] _- 'Groupes analytiques p-adiques', Publ. Math. Inst. Hautes Études Sci. 26 (1965), 389-603.

[7] D. J. S. Robinson, Finiteness conditions and generalized soluble groups (Springer, Berlin, 1972).

[8] P. Shumyatsky, 'On groups with commutators of bounded order', Proc. Amer. Math. Soc. 127 (1999), 2583-2586.

[9] —_ 'Residually finite groups in which commutators are Engel', Comm. Algebra 27 (1999), 1937-1940.

[10] —, 'Applications of Lie ring methods to group theory', in: Nonassociative algebra and its applications (eds. R. Costa, A. Grishkov, H. Guzzo Jr. and L. A. Peresi), Lecture Notes in Pure and Appl. Math. (Marcel Dekker, New York, 2000) pp. 373-395.

[11] J. Tits, 'Free subgroups in linear groups', J. Algebra 20 (1972), 250-270.

[12] J. S. Wilson, 'On the structure of compact torsion groups', Monatshefte für Mathematik 96 (1983), 57-66.

[13] _ 'Two-generator conditions for residually finite groups', Bull. London Math. Soc. 23 (1991), 239-248.

[14] J. S. Wilson and E. I. Zel'manov, 'Identities for Lie algebras of pro-p groups', J. Pure Appl. Algebra 81 (1992), 103-109.

[15] E. I. Zelmanov, 'The solution of the restricted Burnside problem for groups of odd exponent', Math. USSR Izv. 36 (1991), 41-60.

[16] —_, 'The solution of the restricted Burnside problem for 2-groups', Math. Sb. 182 (1991), 568-592.

[17] — 'On periodic compact groups', Israel J. Math. 77 (1992), 83-95.

[18] —, Nil Rings and Periodic Groups, The Korean Math. Soc. Lecture Notes in Math. (Seoul, 1992).

Department of Mathematics

University of Brasilia

70910-900 Brasilia DF

Brazil

e-mail: pavel@ipe.mat.unb.br 